\title{
Programmable Sliceable Transceivers Based on Multicarrier Modulation in Disaggregated Optical Metro Networks
}

\author{
Laia Nadal, Senior Member, IEEE, Josep $M^{a}$ Fabrega, Senior Member, IEEE, Michela Svaluto Moreolo, \\ Senior Member, IEEE \\ Centre Tecnològic de Telecomunicacions de Catalunya (CTTC/CERCA), Castelldefels (Barcelona), Spain \\ Tel: +34 936452 900, Fax: +34 9364529 01, e-mail: laia.nadal@cttc.es
}

\begin{abstract}
The adoption of open network elements (white boxes), based on different technologies, within the disaggregation network paradigm requires the design/implementation of novel adaptive transceiver architectures. We propose a flexible and programmable multi-rate, multi-format, multi-reach modular sliceable bandwith/bitrate variable transceiver (S-BVT) architecture composed of bandwidth/bit rate variable transceiver (BVT) modules, which can be enabled/disabled according to the network needs.

In this paper, we focus on a two-slice architecture of the programmable sliceable transceiver based on multicarrier modulation (MCM), tailored for optical disaggregated metro networks. Thanks to the flexibility and cost-effectiveness of the proposed solution, network dynamicity and elasticity can be supported. Additionally, the effect of traversing multiple network nodes based on different technologies is investigated. Special focus is devoted to the activities and recent results achieved within the H2020 METRO-HAUL project.
\end{abstract}

Keywords: Sliceable bandwidth/bitrate variable transceiver (S-BVT), multicarrier modulation (MCM), filter concatenation, disaggregated optical networks.

\section{INTRODUCTION}

Legacy closed optical networks/systems are composed of network elements/components (such as transceivers, nodes, line amplifiers, etc.), that are designed and optimized by vendors based on a set of proprietary standards, following a vertically-integrated vendor black-box model [1]. Despite this approach provides the ability to abstract functionalities into a single model, network flexibility is very limited. Hence, in a real-life scenario where the number of emerging services, global IP traffic and connected devices is massively increasing, a need for a new networking approach for overcoming vendor lock-in and flexibility limitations is required. Additionally, according to CISCO reporting, the $33 \%$ of total service provider network capacity by 2022 will correspond to the metro segment [2]. This has driven the current metro networking infrastructure toward disaggregation in order to built-in higher adaptability/elasticity and efficiency, whilst reducing cost and power consumption [1][3]. This flexibility, provided by software and hardware disaggregation, will enable to efficiently turn on advanced/new network features and functionalities. In view of an evolution towards this paradigm, different data plane aspects have to be redesigned to provide the scalability pursued by the service providers. To this extend, disruptive efficient, modular and scalable transmission solutions should be investigated. Hence, sliceable bandwidth/bitrate variable transceivers (S-BVTs) arise as a promising solution to introduce system flexibility due to their inherent modularity and capacity to adapt/configure their parameters/elements according to the traffic demand. On the other hand, by the adoption of open and disaggregated models, also new challenges arise such as network interoperability. Hence, open and common data models must be implemented in order to deal with different white box network elements while ensuring a software defined networking (SDN) approach. In this context, new initiatives such as OpenROADM and OpenConfig arise in order to work on the definition and implementation of multisource agreements for optical white boxes [4].

In this paper, a programmable S-BVT based on multicarrier modulation (MCM) and adopting direct detection (DD) receiver configuration is implemented. It is based on the architecture described in [5] and we focus on a two-slice implementation. The proposed (SDN-enabled) S-BVT becomes a major driver towards disaggregation, enabling to bring openness and innovation to the optical metro segment. As a first step, the S-BVT is experimentally assessed. In particular, two different slices are created and aggregated into a single flow of variable capacity. Additionally, a fully disaggregated network scenario based on bare metal blades, where even the subsystems comprising the different network elements (e.g. elements composing nodes) can be from different hardware suppliers, is considered [1]. To that end, different node architectures based on wavelength selective switches (WSS) and arrayed waveguide grating (AWG) technologies are experimentally assessed by using the ADRENALINE testbed, which is a fixed/flexi-grid dense wavelength division multiplexing (DWDM) network with white box reconfigurable optical add-drop multiplexer (ROADM)/ optical cross connect (OXC) nodes [6]. Different network paths have been evaluated in order to consider a multiple-hop scenario with different node architectures. 


\section{PROGRAMMABLE SLICEABLE TRANSCEIVER BASED ON MULTICARRIER MODULATION: ARCHITECTURE AND EXPERIMENTAL SET-UP}
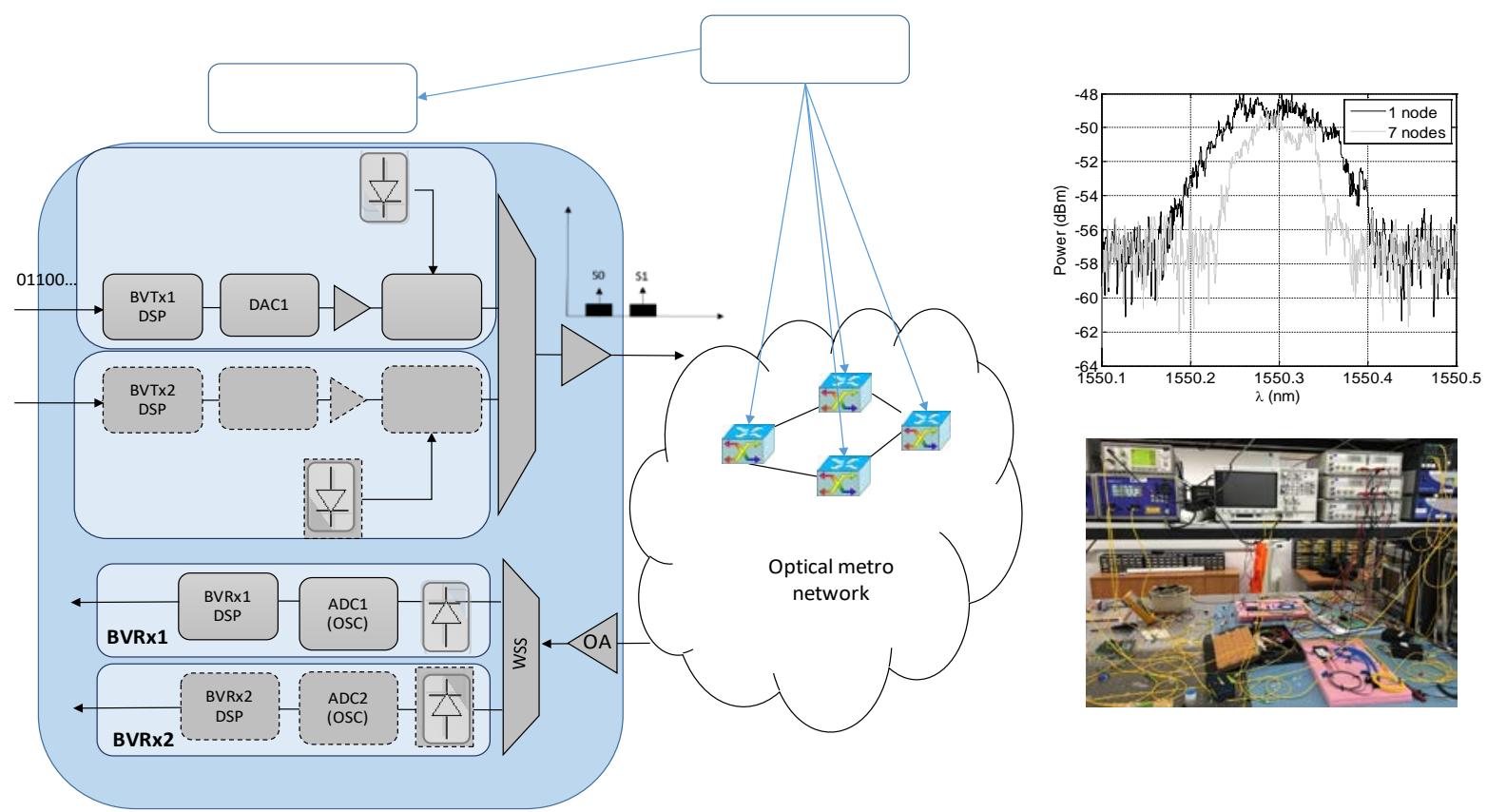

Figure 1. Programmable sliceable transceiver based on multicarrier modulation. In the top-right inset, filter profile of 1 and 7 concatenated nodes based on 25GHz-WSS filters. Each intermediate node has 2 WSS filters. Edge nodes have a single filter. In the bottom-right inset, experimental set-up.

Figure 1 shows the architecture of the proposed programmable multi-rate/format/reach/flow (SDN-enabled) transceiver based on multicarrier modulation (MCM) with receiver configuration adopting direct detection (DD). Two slices, S0 and S1 of Figure 1, can be enabled to transmit a high capacity flow over the network. Additionally, to further increase the system capacity according to the network targets/requirements, the proposed architecture can be upgraded with additional modules in a grow-as-needed approach [7]. The envisioned system architecture reduces the capital expenditure (CapEx) of the network by adopting cost-effective optoelectronic subsystems and simplified digital signal processing (DSP) solutions. Particularly, the optoelectronic front-end of an S-BVT building block consist of a simple Mach-Zehnder modulator (MZM), driven by a tunable laser source (TLS) at the transmitter, and a simple photo-detector (PIN) with transimpedance amplifier (TIA) at the receiver. The two generated flows are aggregated/distributed with a WSS, which serve also as a filter to perform single side modulation (SSB) at the transmitter side. SSB-OFDM implementation increases system resilience towards chromatic dispersion, enabling to achieve extended reach and higher data rates.

Digital-to-analogue conversion (DAC) is performed at $64 \mathrm{GSa} / \mathrm{s}$; while the analogue-to-digital conversion (ADC) is performed, for the experimental assessment, using a digital oscilloscope (OSC) with sample rate of 100 GSa/s. Thanks to the implementation of discrete multitone (DMT) / orthogonal frequency division multiplexing (OFDM) modulation, subcarrier granularity is achieved, enabling to dynamically adapt the transmission according to the network demand. At the DSP block of the transmitter, randomly generated data is mapped into different constellations such as BPSK and M-QAM (with $M=2^{n}$ and $2 \leq n \leq 8$ ) according to the channel profile. Specifically, bit and power loading algorithms (BL/PL) can be implemented to optimize the modulation format and power value per subcarrier [8]. After adding the training symbols (TS), the inverse fast Fourier transform (IFFT) is implemented and a cyclic prefix (CP) is appended to perform the DMT/OFDM modulation. At the receiver side, CP removal, FFT implementation, equalization and demaping processes take place. The overheads due to CP and TS are $1.9 \%$ and 4\%, respectively. A hard decision (HD) forward error correction (FEC) of $7 \%$, corresponding to a bit error rate (BER) of $4.62 \cdot 10^{-3}$, is considered. The BER is calculated by error counting.

Operational expenditure (OpEx) is also reduced, by implementing the corresponding SDN agents to configure/program the S-BVT according the network requirements/needs for an efficient use of the metro transport resources. With the adoption of the SDN paradigm, the transceiver flexibility can be fully exploited, allowing a dynamic management of optical networks.

\section{ON S-BVT PROGRAMMABILITY AND RECONFIGURABILITY}

The programmability and reconfigurability of the S-BVT has a key role towards the integration of the data and control planes to support advanced functionalities, such as slice-ability and rate/distance and bandwidth adaptability. Hence, a set of reconfigurable parameters and programmable elements have been identified. 
Specifically, different system parameters such as the bandwidth occupancy, FEC, loading algorithm selection, MZM bias and equalization type can also be modified/reconfigured to fully exploit the system flexibility enhancing its performance, as detailed in [5]. Here, SDN agents are implemented following the OpenConfig model. Hence, the set of reconfigurable parameters is reduced to TLS central wavelength, output power and operational mode with the aim to move toward a more dynamic, programmable method for configuring and managing multivendor networks. The operational mode field is vendor-specific and provides different channel mode/configuration to be selected, such as modulation, symbol rate and FEC. FEC mode can be interesting in the case of adopting programmable S-BVTs based on MCM to set the target performance as an input of a loading algorithm, which can be applied, as an advanced functionality, to maximize the system capacity. Regarding the programmable elements, the DSP, the TLS (central wavelength, power), the WSS (bandwidth, central wavelength, phase and attenuation per port) and the optical amplifier (power and operational mode), can be programmed by the SDN controller through SDN agents according to the network needs/specifications. Each S-BVT, which can be seen as an Openconfig terminal device, has its own OpenConfig agent that configures the different modules/building blocks [4]. Finally, specific key performance indicators (KPIs) have been also identified in order to evaluate/measure with different metrics the proposed transceiver performance (see Table 1). This includes, slice-ability functionality, BER, capacity, bit rate variability and programmability. In particular 2 slices are enabled and experimentally assessed in section 4 . A target capacity of $50 \mathrm{~Gb} / \mathrm{s}$ is experimentally demonstrated at $4.62 \cdot 10^{-3} \mathrm{BER}$. Bit rate variability is also investigated by applying BL/PL algorithms. Finally, SBVT programmability is enabled by the adoption of SDN agents.

Table 1. S-BVT key performance indicators.

\begin{tabular}{|c|c|c|}
\hline KPI & Target specifications & Means of verification \\
\hline Slice-ability & 2 slices & Number of slices \\
\hline BER & $\begin{array}{l}\text { HD-FEC }\left(4.62 \cdot 10^{-3}\right) \\
\text { SD-FEC }\left(2 \cdot 10^{-3}\right)\end{array}$ & Error counting \\
\hline Capacity & $50 \mathrm{~Gb} / \mathrm{s}$ (B2B) per slice & $\begin{array}{l}\text { BW*bps (bandwidth occupancy per bit } \\
\text { per symbol }\end{array}$ \\
\hline Bit rate variability & $10 \mathrm{~Gb} / \mathrm{s}-50 \mathrm{~Gb} / \mathrm{s}$ per slice & Subcarrier granularity (slice BW/subc) \\
\hline Programmability (Openconfig model) & $\begin{array}{l}\text { Laser central wavelength (TLS) } \\
\text { Laser output power } \\
\text { Operational mode }\end{array}$ & $\begin{array}{l}\text { SDN agents controlling DSP, TLS, WSS } \\
\text { and OA }\end{array}$ \\
\hline
\end{tabular}

\section{PERFORMANCE IN DISAGGREGATED OPTICAL NETWORKS}

In this section, the proposed S-BVT has been experimentally assessed in back-to back (B2B) and over different fixed/flexi-grid paths of the ADRENALINE testbed. A first level of disaggregation is demonstrated in B2B configuration, by considering an S-BVT with white box building blocks. For this purpose, two slices (S0 and S1), generated with components from different manufacturers, are transmitted at $1550.14 \mathrm{~nm}$ and $1550.5 \mathrm{~nm}$ central wavelength, corresponding to channels C34 and H33 of the ITU-T 50GHz grid (see Fig. 1). According to Fig. 2 (a), an aggregated data flow of $100 \mathrm{~Gb} / \mathrm{s}$ can be transmitted with uniform loading (UL) at the target BER. Specifically, S0 supports $60 \mathrm{~Gb} / \mathrm{s}$ B2B data rate; while S1 achieves $40 \mathrm{~Gb} / \mathrm{s}$ due to hardware limitations. Specifically, the two slices are created with different bandwidth variable transceivers (BVTs) from different providers. This capacity can be further enhanced by implementing BL/PL algorithms, which can adapt the number of bits/power values per subcarrier according to the channel profile [8]. In fact, an aggregated flow of about $125 \mathrm{~Gb} / \mathrm{s}$ is supported with BL/PL with $44 \mathrm{~dB}$ optical signal-to-noise ratio (OSNR), as shown in Fig. 2 (b).

A second approach towards a fully disaggregated model based on bare metal blades is assessed considering the ADRENALINE white box ROADM/OXC nodes [1,3]. Specifically, two different network node architectures are investigated based on WSS and AWG technologies. Additionally, a module emulating up to 7 cascaded nodes based on WSS is included in the experiment in order to evaluate the impact of the filter narrowing effect on the system performance [9]. A single filtering stage is considered to add/drop the different signals at the edge nodes. Conversely, at intermediate network nodes, 2 filters are included. In these networking scenarios, the implementation of BL/PL algorithms are crucial in order to compensate the effects of the filtering. Figure 2 (c), reports the performance, in terms of data rate, of a single slice (S0) after traversing multiple network white box nodes. In particular, S0 supports $60 \mathrm{~Gb} / \mathrm{s}$, with BL/PL, after 7 cascaded nodes based on WSS filter of $50 \mathrm{GHz}$ bandwidth. Considering a narrower filter bandwidth $(25 \mathrm{GHz})$, the data rate decreases to $39 \mathrm{~Gb} / \mathrm{s}$, due to the filter narrowing effect. In fact, in the inset of Fig. 1 it can be seen the effect, in terms of bandwidth reduction, of traversing up to 7 nodes. Then, in addition to the cascading module, the flexi-grid ADRENALINE path of 35km is assessed fixing a channel grid of $50 \mathrm{GHz}$ and $25 \mathrm{GHz}$. The achieved rate per slice is $50 \mathrm{~Gb} / \mathrm{s}$ and $31 \mathrm{~Gb} / \mathrm{s}$, respectively. Hybrid approaches, combining WSS and AWG network node technologies, are also investigated in line with the fully disaggregation concept. Thus, the 2-hop and 3-hop ADRENALINE paths of $70 \mathrm{~km}$ and 120 $\mathrm{km}$, are evaluated. In the longest path scenario, up to $36 \mathrm{~Gb} / \mathrm{s}$ per slice can be supported considering up to 10 concatenated nodes. By including narrower filtering in the nodes, this data rate decreases of $6 \mathrm{~Gb} / \mathrm{s}$. 


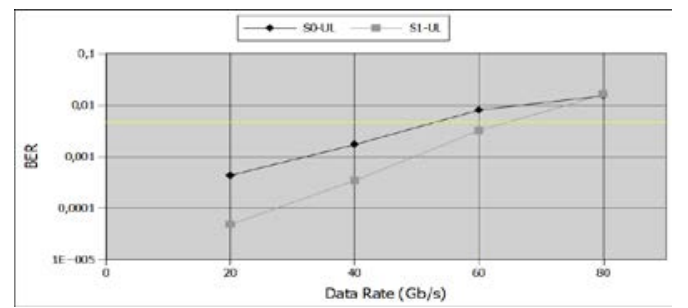

(a)

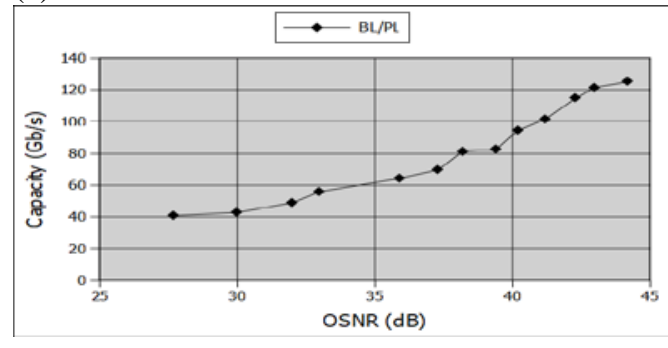

(c)

\begin{tabular}{|l|l|l|l|l|}
\hline Scenario & $\begin{array}{l}\text { Node } \\
\text { technology }\end{array}$ & \# of nodes & Filter BW (GHz) & Data rate (Gb/s) \\
\hline B2B & WSS & 7 & 50 & 60 \\
\hline B2B & WSS & 7 & 25 & 39 \\
\hline 35 km ADRENALINE & WSS & 8 & 50 & 50 \\
\hline 35 km ADRENALINE & WSS & 8 & 25 & 31 \\
\hline $\begin{array}{l}\text { 70 km 2-hop path } \\
\text { ADRENALINE }\end{array}$ & $\begin{array}{l}\text { Hybrid } \\
\text { WSS+AWG }\end{array}$ & 9 & 50 & 46 \\
\hline $\begin{array}{l}\text { 70 km 2-hop path } \\
\text { ADRENALINE }\end{array}$ & $\begin{array}{l}\text { Hybrid } \\
\text { WSS+AWG }\end{array}$ & 9 & 25 & 35 \\
\hline $\begin{array}{l}\text { 120 km 3-hop path } \\
\text { ADRENALINE }\end{array}$ & $\begin{array}{l}\text { Hybrid } \\
\text { WSS+AWG }\end{array}$ & 10 & 50 & 36 \\
\hline $\begin{array}{l}\text { 120 km 3-hop path } \\
\text { ADRENALINE }\end{array}$ & $\begin{array}{l}\text { Hybrid } \\
\text { WSS+AWG }\end{array}$ & 10 & 25 & 30 \\
\hline
\end{tabular}

(b)

Figure 2. (a) B2B UL performance with SO and S1 in terms of data rate vs BER. (b) Aggregated capacity vs OSNR with BL/PL. (c) SO performance after traversing different nodes of a disaggregated optical network.

\section{CONCLUSIONS}

Hardware and software disaggregation arises as a common strategy to enhance network efficiency and reduce the overall cost and power consumption, enabling network-wide programmability/flexibility and observability. In this context, multi-vendor programmable sliceable transceivers, based on open white box sub-transceivers, can be adopted to provide a high degree of reconfigurability, scalability and flexibility to the network. Thanks to the implementation of SDN agents based on the OpenConfig vendor-neutral model, the proposed S-BVT can be suitably programmed and reconfigured according to the network condition/requirements. Recent results assessing a fully disaggregated scenario/network based on bare metal blades have been here reported. $36 \mathrm{~Gb} / \mathrm{s}$ capacity per slice is supported after traversing up to 10 nodes with different architectures. Considering nodes with narrower filtering, this data rate is reduced to $30 \mathrm{~Gb} / \mathrm{s}$.

\section{ACKNOWLEDGEMENTS}

This work has been supported by the EU-H2020 Metro-Haul Project with G.A 761727 and the Spanish AURORAS project (RTI2018-099178). We would like to thank Aragon Photonics for providing the high resolution OSA equipment.

\section{REFERENCES}

[1] Riccardi, E., Gunning, P, González de Dios, et al., 'An Operator view on the Introduction of White Boxes into Optical Networks', J. Lightwave Technol, vol. 36, n. 15, pp. 3062-3072, 2018.

[2] CISCO White Paper: “Cisco Visual Networking Index: Forecast and Trends, 2017-2022”, February 2019.

[3] De Leenheer, M., Tofigh, T., and Parulkar, G., 'Open and programmable metro networks', in 2016 Optical Fiber Communications Conf. and Exhibition (OFC), paper Th1A.7, March 2016.

[4] OpenConfig web site, Mar. 2018. [Online]. Available: http://www.openconfig.net/

[5] M. Svaluto Moreolo, J. M. Fabrega, L. Nadal, L. Martin, 'Optical Technology Options for Programmable S-BVT’, ICTON, 1-5 July, 2018, Bucharest (Romania).

[6] Muñoz, R., Nadal, L., Casellas, R., et al., 'The ADRENALINE Testbed: An SDN/NFV Packet/Optical Transport Network and Edge/Core Cloud Platform for End-to-End 5G and IoT Services', Proc. of European Conf. on Netw. and Comm. (EUCNC), Oulu, Finand, June 2017.

[7] Svaluto Moreolo, M., Fàbrega, J.M., Nadal, L., et al., 'SDN-enabled Sliceable BVT Based on Multicarrier Technology for Multi-Flow Rate/Distance and Grid Adaptation', J. Lightwave Technol, vol. 34, n. 6, pp. 1516-1522, 2016.

[8] Nadal, L., Svaluto Moreolo, M., Fàbrega, J. M., et al., 'DMT Modulation with Adaptive Loading for High Bit Rate Transmission Over Directly Detected Optical Channels ', Journal of Lightwave Technology, 2014, 32, (21), pp. 3541-3551.

[9] Fàbrega, J.M., Svaluto Moreolo, M., Martín, L., et al., "On the filter narrowing issues in elastic optical networks," J. of Optical Communications and Networking, Vol. 8, No. 7, pp. A23-A33, July 2016. 